\title{
Entraînement d'air dans les écoulements à surface libre : application aux évacuateurs de crues de barrage
}

\author{
Hubert Chanson \\ Maître de Conférences \\ Department of Civil Engineering, The University of Queensland \\ (St Lucia QLD 4072, Australia)
}

\section{Présentation}

\subsection{Introduction}

Pour les écoulements à surface libre, la quantité d'air entraînée est un paramètre important pour le dimensionnement des canaux. L'air augmente le volume de l'écoulement, par diminution de la masse spécifique, et ceci doit être pris en compte pour le calcul de la hauteur des murs latéraux (FALVEY 1980).

En outre, la présence de bulles d'air dans la couche limite turbulente diminue les contraintes de cisaillement entre les filets de liquide, et donc les forces de frottement (Wood 1983). Il en résulte une augmentation de la quantité de mouvement qui doit être prise en compte pour le calcul des tremplins (ski jump) et la location des bassins de dissipation (stilling basin).

\begin{abstract}
De plus, la présence d’air dans les écoulements à hautes vitesses permet de ralentir ou de prévenir les dommages dus à l'érosion par cavitation (MAY 1987). Il est nécessaire de connaître la quantité d'air entraînée au long du coursier, pour estimer l'emplacement optimal des dispositifs d'aération, ou déterminer le besoin d'installer, ou non, ces dispositifs d'aération (CHANSON 1989).

Plus récemment, le rôle de l'entraînement d'air dans les écoulements à surface libre a été reconnu dans le cadre des transferts d'oxygène et d'azote entre l'air et l'eau, dans les écoulements (WILHELMS et Gulliver 1989). La présence d'air dans les écoulements à surface libre, augmente le transfert de gaz atmosphériques dans les rivières et canaux : ceci peut être utilisé pour réoxygéner des cours d'eau pollués, mais aussi explique le taux de mortalité important des poissons, en aval de certains grands ouvrages hydrauliques.
\end{abstract}

\section{Air entrainment in open channel flows} Application to spillways

In open channel flows an important design parameter is the amount of entrained air. The presence of air in high velocity flows or steep chutes increases the bulk of the flow and this is a design pardmeter that determines the height of chute sidewalls. Furthermore the presence of air within the boundary layer reduces the shear stress between flow layers and hence the shear forces. The resulting increase of fluid motion must be taken in account when designing ski jump and stilling basin downstream of a spillway. Besides the presence of air within high-velocity flows may prevent or reduce cavitation damage. Estimation of the quantity of air entrained along the spillway is required by the design engineer when sitting aerators to artificially introduce more air, or determining whether aerators are required. Recently air entrainment on chutes also been recognized for its contribution to air-water transfer of atmospheric gases such as oxygen and nitrogen.

This article describes a general method to compute the flow characteristics of self-aerated flows. Firstly the uniform flow conditions are presented and comparisons will be made between model and prototype results. Then the basic equations for gradually varied aerated flows will be developed, and these results are discussed and compared with experimental data. 


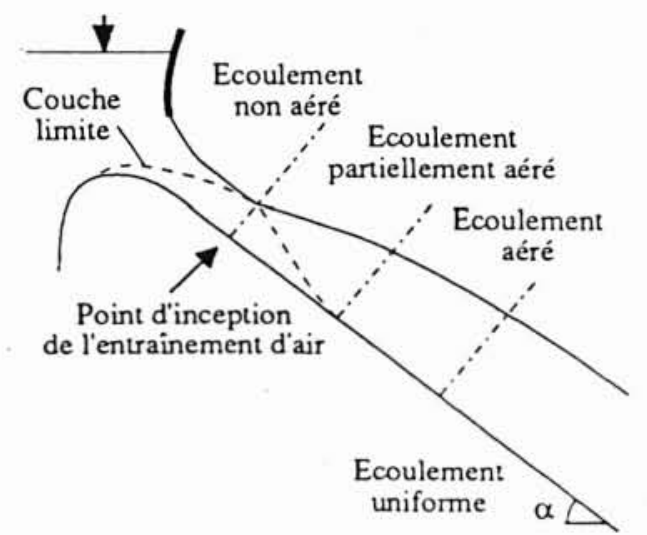

1. Entraînement d'air dans un écoulement à surface libre sur un coursier d'évacuateur de crues de barrage.

\subsection{Entraînement d'air dans un écoulement à surface libre}

Considérant un écoulement sur un coursier d'évacuateur de crues, à l'amont, une couche limite turbulente se développe au long du coursier, et elle atteint la surface libre au point d'inception (inception point), marquant le début de la zone d'écoulement aéré (fig. 1). La position de ce point se déduit en utilisant les abaques de KELLER et RATOGi (1977), FALVEY (1980) ou Wood (1985).

En aval du point d'inception, l'accroissement de la quantité d'air entraînée est graduel et continu. Les profils de concentrations en air et de vitesses se redistribuent lentement (région d'écoulement graduellement varié), et tendent vers des profils d'équilibre dans la région d'écoulement uniforme, loin en aval (WooD 1983).

\subsection{Mécanismes d'entraînement d'air}

Pour des écoulements supercritiques, à forte pente, une grande quantité d'air est entraînée à la surface libre, par une multitude de vortex turbulents et irréguliers, proches de la surface libre (Wood 1985). Des bulles et poches d'air sont entraînées sous l'action de la vitesse turbulente perpendiculaire à la surface libre. Et à travers l'interface eau-air, il y a constamment un échange de gaz, s'échappant vers l'atmosphère, sous l'effet des forces d'Archimède, ou entraîné sous l'action de la turbulence.

\subsection{Définitions}

La concentration en air locale $C$ est définie comme le volume d'air par unité de volume. On définit la profondeur de référence $d$ comme :

$$
d=\int_{C=0}^{C=90 \%}(1-C) * \mathrm{~d} y
$$

où $y$ est mesuré perpendiculairement à la surface du coursier. On définit une autre profondeur de référence $Y_{90}$ comme celle où $C=90 \%$. La concentration en air moyenne $C_{\text {mean }}$, dans une section transversale à l'écoulement, est définie comme :

$$
\left(1-C_{\text {mean }}\right) * Y_{90}=d .
$$

La vitesse moyenne $U_{w}$ de l'écoulement, dans une section, est :

$$
U_{w}=\frac{q_{w}}{d}
$$

et une autre vitesse de référence $\left(V_{90}\right)$ est définie comme celle pour $y=Y_{90}$.

\section{Ecoulements uniformes}

\subsection{Concentration en air}

\subsubsection{Distribution de la concentration en air}

WOOD (1983) analysa les mesures expérimentales obtenues par Straub et ANDERSON (1958), pour des écoulements uniformes, et montra que les profils de distribution de la concentration en air, sous la forme adimensionnelle $C=f\left(y / Y_{90}\right)$ (fig. 2), sont indépendants des conditions amont (débit, profondeur, rugosité), et sont fonction de la pente du canal, uniquement.

De plus, Wood (1984) développa un modèle simple pour représenter la diffusion de bulles d'air dans un écoulement turbulent, et montra que la distribution de la concentration en air s'exprime :

$$
C=\frac{B^{\prime}}{B^{\prime}+\mathrm{e}^{-\left(G^{\prime} \cdot \cos \alpha * y^{\prime 2}\right)}}
$$

où $B^{\prime}$ and $G^{\prime}$ sont des constantes, fonction de la pente (tabl. l), et $y^{\prime}$ est la profondeur adimensionnelle : $y^{\prime}=y / Y_{90}$.

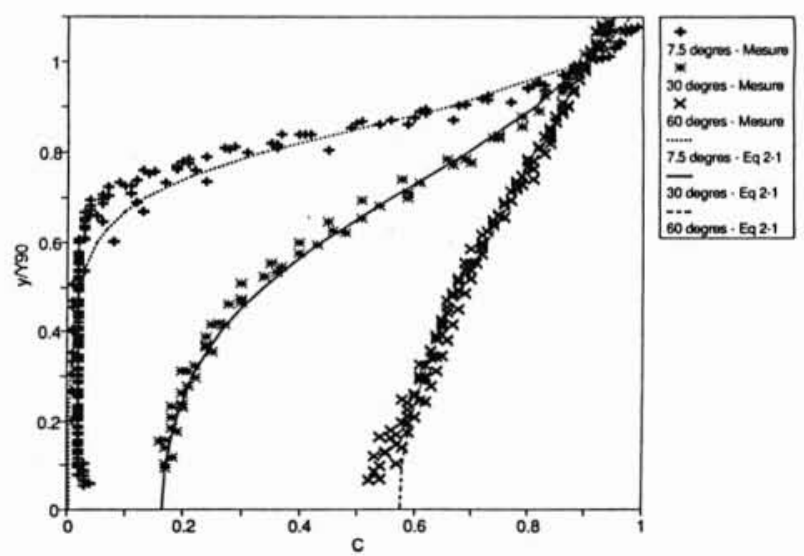

2. Profils de concentrations en air pour trois pentes $(7,5,30 \mathrm{et}$ $\left.60^{\circ}\right)$, avec des débits compris entre 0,136 et $0,595 \mathrm{~m}^{2} / \mathrm{s}-$ Straub et ANDERSON (1958). 


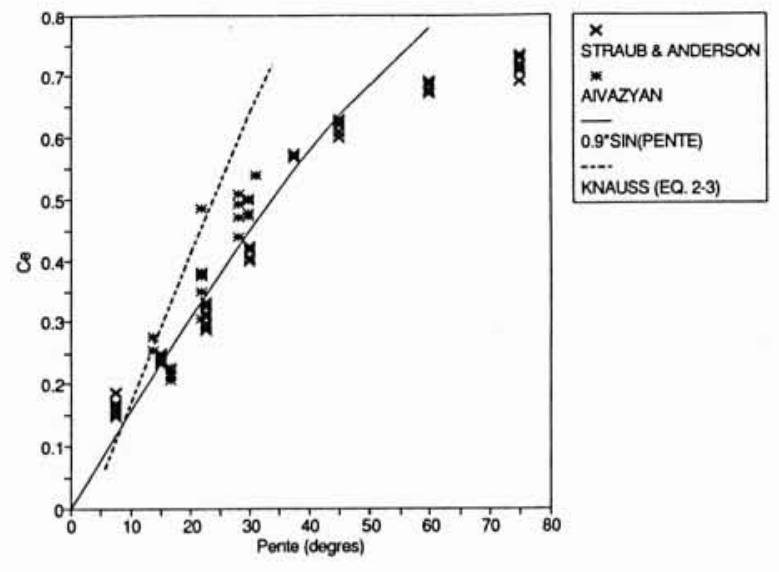

3. Concentration en air moyenne pour les écoulements uniformes $C_{e}$ en fonction de la pente du canal $\alpha$ - Straub et ANDERSON (1958) - AIVAZYAN (1986) - KNAUSS (1979).

\subsubsection{Concentration en air moyenne}

Une analyse détaillée des mesures obtenues par STRAuB et ANDERSON, montre que la concentration en air moyenne pour les écoulements uniformes $C_{e}$, est indépendante du débit et du nombre de Froude (WoOD 1985 ; Chanson 1989), et que $C_{e}$ est une fonction de la pente du canal uniquement (tabl. 1). La figure 3 présente les résultats déduits des mesures de STRAub et ANDERSON (1958) et de AIVAZYAN (1986). Et on en déduit une relation simple entre la pente $\alpha$ et la concentration moyenne en air :

$$
C_{e}=0,9 * \sin \alpha \quad \text { pour } \quad \alpha<50^{\circ} .
$$

\section{Discussion}

HARTUNG et Scheuerlein (1970) ont effectué des mesures sur des écoulements avec des rugosités importantes ( $k_{s}$ entre 0,1 et $\left.0,35 \mathrm{~m}\right)$, et avec des pentes de 6 à $34^{\circ}$. Ces rugosités importantes induisent un écoulement fortement turbulent, avec entraînement d'air, et KNAUSS (1979) indique que le taux d'aération, exprimé en terme de concentration en air moyenne, est :

$$
C_{e}=1,44 * \sin \alpha-0,08 .
$$

Cette formule est très proche des résultats ci-dessus (équation (2.2)), et est reportée sur la figure 3.

\subsection{Profil de vitesses}

CAIN (1978) mesura des profils de vitesses, sur l'évacuateur de crues du barrage d'Aviemore (NouvelleZélande), avec une pente de $45^{\circ}$, et des concentrations en air moyennes variant de 0 à $50 \%$ (fig. 4). CAIN et WoOD (1981) ont montré que la distribution des vitesses peut être écrite sous forme adimensionnelle :

$$
\frac{V}{V_{90}}=\left(\frac{y}{Y_{90}}\right)^{1 / 6}
$$

où l'exposant $(1 / 6,0)$ a été recalculé par l'auteur (CHANSON 1989) à partir des mesures expérimentales (CAIN 1978). Bien que ces mesures aient été obtenues pour des écoulements graduellement variés, il est clair, que le profil des vitesses est quasiment indépendant de la concentration en air moyenne, et il est raisonnable de supposer que ce résultat s'applique aussi aux écoulements uniformes.

La vitesse de référence $V_{90}$ se déduit, à partir de l'équation de conservation de la masse (pour l'eau), en utilisant l'équation (2.4) :

$$
V_{90}=\frac{q_{w}}{Y_{90}} *\left(\int_{C=0}^{C=90 \%}(1-C) * y^{\prime 1 / 6} * \mathrm{~d} y^{\prime}\right)^{-1}
$$

où $C$ est calculée en utilisant l'équation (2.1) et $y^{\prime}=y / Y_{90}$.

De même, on définit un coefficient de correction de quantité de mouvement $M$, et un coefficient de correction d'énergie cinétique $\mathrm{E}$ (coefficient de Coriolis), pour les écoulements aérés :

$$
\begin{gathered}
M=\left(1-C_{e}\right) * \frac{\int_{C=0}^{C=90 \%}(1-C) * y^{\prime 2 / 6} * \mathrm{~d} y^{\prime}}{\left(\int_{C=0}^{C=90 \%}(1-C) * y^{\prime 1 / 6} * \mathrm{~d} y^{\prime}\right)^{2}} \\
E=\left(1-C_{e}\right)^{2} * \frac{\int_{C=0}^{C=90 \%}(1-C) * y^{\prime 3 / 6} * \mathrm{~d} y^{\prime}}{\left(\int_{C=0}^{C=90 \%}(1-C) * y^{\prime 1 / 6} * \mathrm{~d} y^{\prime}\right)^{3}} .
\end{gathered}
$$

Les équations (2.5), (2.6) et (2.7) ont été calculées, pour différentes pentes ou concentrations en air moyennes (tabl. 1).

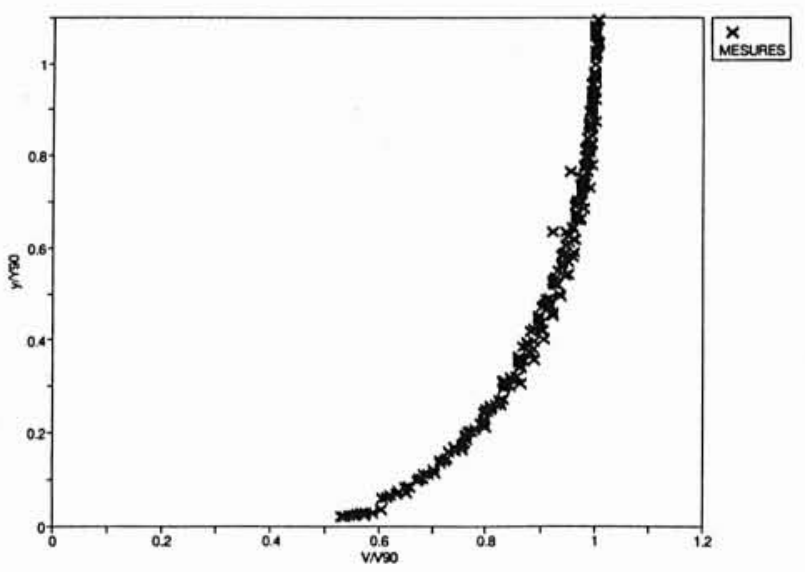

4. Profil des vitesses mesurées sur le coursier de l'évacuateur de crues d'Aviemore - CAIN (1978) - Stations 501, 502, 503, 504 et $505-q_{w}=2,23 \mathrm{~m}^{2} / \mathrm{s}$ et $3,16 \mathrm{~m}^{2} / \mathrm{s}$. 


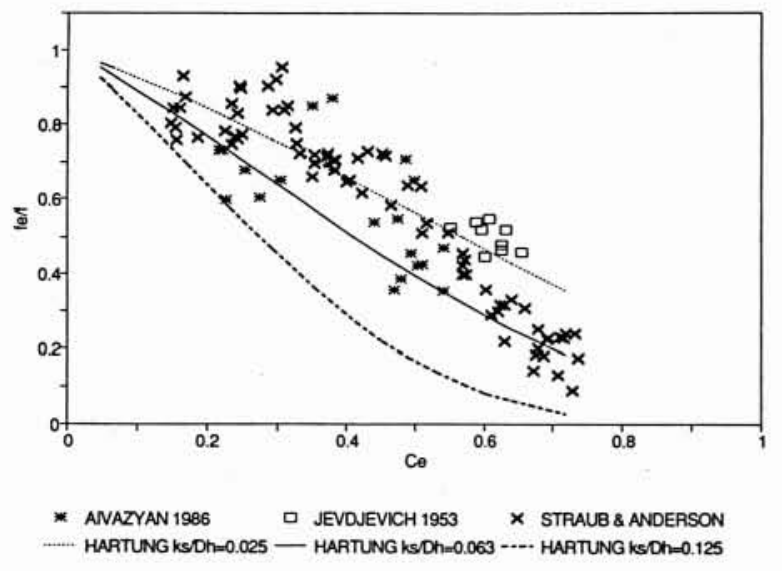

5. Coefficient de perte de charge relatif $f_{e} / f$ en fonction de la concentration en air moyenne $C_{e}$ - JEVDJEVICH et LEVIN (1953) - Straub et Anderson (1958) - Aivazyan (1986).

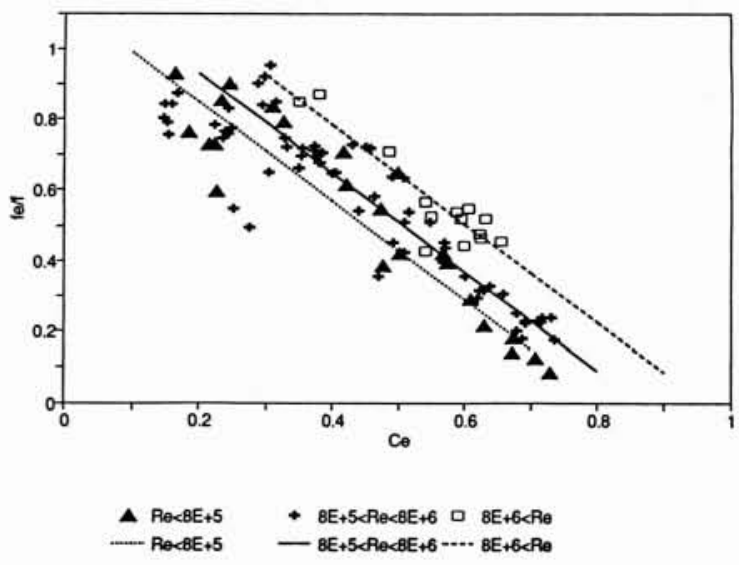

6. Corrélation entre le coefficient de perte de charge, la concentration en air moyenne et le nombre de Reynolds JeVDjeVich et LeVIN (1953) - Straub et Anderson (1958) - Aivazyan (1986).

\subsection{Coefficients de perte de charge}

\subsubsection{Coefficient de perte de charge}

La présence d'air, dans l'écoulement, affecte peu le profil des vitesses, mais KILLEN (1968) et WoOD (1983) ont suggéré que la présence de bulles et poches d'air, dans la couche limite turbulente, entraîne une réduction des contraintes de cisaillement entre les filets de liquide.

Considérant un écoulement uniforme, l'équation de conservation de l'énergie s'écrit :

$$
f_{e}=\frac{8 * g * \sin \alpha * d^{2}}{q_{w}^{2}} *\left(\frac{D_{H}}{4}\right)
$$

où $f_{e}$ est le coefficient de perte de charge pour un écoulement uniforme aéré, $d$ est la profondeur de référence (équation (1.1)), et $D_{H}$ est le diamètre hydraulique.

L'auteur a analysé les mesures obtenues par JEVDJEVICH et Levin (1953), Straub et ANDERson (1958) et AIVAZYAN (1986), utilisant l'équation (2.8), et les résultats montrent une réduction importante du coefficient de perte de charge quand la concentration en air augmente (fig. 5).

Une analyse adimensionnelle du coefficient de perte de charge indique que le rapport $f_{e} / f$, où $f$ est le coefficient de perte de charge d'un écoulement non-aéré, est fonction de la concentration en air moyenne, du nombre de Reynolds et de la rugosité :

$$
\frac{f_{e}}{f}=\Phi\left(C_{e} ; \operatorname{Re} ; \frac{k_{s}}{D_{H}}\right) .
$$

\subsubsection{Influence du nombre de Reynolds}

Pour les mesures expérimentales obtenues par JEVDJEVich et Levin (1953), Straub et ANDERson (1958) et
Aivazyan (1986), l'équation (2.9) peut être ré-écrite comme :

$\frac{f_{e}}{f}=0,307+0,1446 * \log _{10}(\operatorname{Re})-1,40 * C_{\text {mean }}$

pour $C_{e}>0,25$, et des nombres de Reynolds compris entre $2.10^{5}$ et $4.10^{7}$. La figure 6 suggère que le rapport $f_{e} / f$ tend vers l'unité, quand le nombre de Reynolds augmente.

Lorsque le nombre de Reynolds augmente, les contraintes de cisaillement entre les filets de liquide augmentent, et la taille maximale des bulles d'air diminue. Il est raisonnable de penser que la turbulence est moins affectée par des bulles de petites tailles, et ceci pourrait expliquer que $f_{e}$ tend vers $f$ lorsque le nombre de Reynolds augmente.

\subsubsection{Influence de la rugosité}

Une analyse détaillée de ces mesures indique que le rapport $f_{e} / f$ depend peu de la rugosité, pour ces expérimentations : $5 \cdot 10^{-4}<k_{s} / D_{H}<5 \cdot 10^{-2}$. Dans cet intervalle, l'effet de la rugosité sur le coefficient de perte de charge est le même avec ou sans entraînement d'air.

Toutefois, ce résultat doit être complété par les mesures faites par HARTUNG et SCHEUERLEIN (1970), avec des canaux à grande rugosité $\left(0,02<k_{s} / D_{H}<0,2\right)$, et leurs résultats fournissent la relation suivante :

$$
\frac{1}{\sqrt{f_{e}}}=\frac{1}{\sqrt{f}}-3,2 * \log _{10}\left(1-C_{e}\right) .
$$

Cette équation est proche des résultats précédents, mais indique que, pour une concentration en air moyenne donnée, le rapport $f_{e} / f$ décroît lorsque la rugosité augmente (fig. 5). Cependant, ce résultat ne s'applique que pour des rugosités importantes. 


\section{ENTRAÎNEMENT D'AIR DANS LES ÉCOULEMENTS À SURFACE LIBRE}

\subsubsection{Discussion}

Une réduction similaire du coefficient de perte de charge a aussi été observée pour des écoulements avec des particules en suspension. VANONI (1946) suggère que la présence de matériaux en suspension réduit l'intensité de la turbulence, et, avec des particules de même densité que l'eau, ElATA et IPPEN (1961) ont montré que la présence de particules en suspension modifie la structure de la turbulence.

Certaines mesures, avec des matériaux en suspension (VANONI 1946), ou des écoulements aérés (KILLEN 1968), indiquent que la constante universelle de Von Karman $K$, caractérisant le profil logarithmique de vitesses, diminue lorsque la concentration en particules augmente. Mais RAO et KoBUS (1971) montrent que la présence de bulles d'air augmente la valeur de cette constante, tandis que COLEMAN (1981) indique que cette constante universelle n'est en rien affectée par la présence de particules. Les mécanismes d'interaction entre la turbulence et les particules en suspension sont mal compris, mais il est certain que les propriétés de la turbulence sont affectées par la présence de particules en suspension.

\subsection{Caractéristiques des écoulements uniformes}

Pour un écoulement uniforme avec entraînement d'air, la géométrie du coursier (pente, rugosité, largeur) et le débit permettent de calculer toutes les caractéristiques de l'écoulement. A partir de la pente du coursier $\alpha$, la figure 3 donne la concentration en air moyenne $C_{e}$, et connaissant le coefficient de perte de charge $f$, la figure 5 fournit la valeur de $f_{e}$. La profondeur de référence $d$ est déduite de l'équation (2.8), et pour un canal de grande largeur, on obtient :

$$
d=\left(\frac{q_{w}^{2} * f_{e}}{8 * g * \sin \alpha}\right)^{1 / 3} .
$$

On déduit alors: $1^{\circ}$ la profondeur caractéristique $Y_{90}$ (équation (1.2)), $2^{\circ}$ la vitesse de référence $V_{90}$ (équation (2.5)), et $3^{\circ}$ les profils de concentrations en air (équation (2.1)) et de vitesses (équation (2.4)).

\section{Ecoulements graduellement variés}

\subsection{Equation de conservation de la masse pour l'air}

L'équation de conservation de la masse, pour l'air entraîné dans l'écoulement, prend en compte l'action des fluctuations de vitesses turbulentes, et des forces d'Archimède, et elle s'écrit (WOOD 1985) :

$$
\frac{\mathrm{d}}{\mathrm{d} s} q_{\mathrm{air}}=V_{\mathrm{e}}-C_{\text {mean }} * u_{r} * \cos \alpha
$$

où $V_{e}$ est la vitesse d'entraînement d'air, caractérisant l'action de la turbulence, et $u_{r}$ est la vitesse limite d'ascension des bulles d'air. Pour un écoulement uniforme, la limite de l'équation (3.1) est :

$$
0=\left[V_{e}\right]_{e}-C_{e} *\left[u_{r}\right]_{e} * \cos \alpha
$$

où $\left[V_{e}\right]_{e},\left[u_{r}\right]_{e}$ et $C_{e}$ sont la vitesse d'entraînement d'air, la vitesse limite d'ascension des bulles d'air, et la concentration en air moyenne, pour un écoulement uniforme.

En supposant que les vitesses d'entraînement et d'ascension $V_{e}$ et $u_{r}$ sont identiques, pour les écoulements graduellement variés comme pour les écoulements uniformes, on obtient :

$$
\begin{aligned}
\frac{\mathrm{d}}{\mathrm{d} s^{\prime}} C_{\text {mean }}= & \left(1-C_{\text {mean }}\right) \times \\
& \times\left[\frac{u_{r} * d_{.} * \cos \alpha}{q_{w}} *\left(C_{e}-C_{\text {mean }}\right) \times\right. \\
& \left.\times\left(1-C_{\text {mean }}\right)+\frac{C_{\text {mean }}}{W^{\prime}} * \frac{\mathrm{d} W^{\prime}}{\mathrm{d} s^{\prime}}\right]
\end{aligned}
$$

où $C_{e}$ est fonction de la pente du coursier, $d$. est la profondeur de référence à l'origine $(s=0), s^{\prime}=s / d$, et $W^{\prime}=W / d$. L'équation (3.2) permet de calculer, en tout point, la concentration en air moyenne, indépendamment de la vitesse, de la rugosité et de la profondeur.

Pour un canal de largeur constante, l'équation (3.2) se simplifie :

$$
\frac{\mathrm{d}}{\mathrm{d} s^{\prime}} C_{\text {mean }}=\frac{u_{r} * d_{*} * \cos \alpha}{q_{w}} *\left(C_{e}-C_{\text {mean }}\right) *\left(1-C_{\text {mean }}\right)^{2}
$$

et, pour un canal à pente constante, la solution analytique de cette équation est :

$$
\begin{aligned}
\frac{1}{\left(1-C_{e}\right)^{2}} & \operatorname{Ln}\left(\frac{1-C_{\text {mean }}}{C_{e}-C_{\text {mean }}}\right)- \\
& -\frac{1}{\left(1-C_{e}\right) *\left(1-C_{\text {mean }}\right)}=k * s^{\prime}+K_{0}
\end{aligned}
$$

et $K_{0}$, constante d'intégration, et $k$ sont respectivement :

$$
\begin{aligned}
K_{0} & =\frac{1}{1-C_{e}} *\left(\frac{1}{1-C_{e}} * \operatorname{Ln}\left(\frac{1-C_{.}}{C_{e}-C_{.}}\right)-\frac{1}{1-C_{.}}\right) \\
k & =\frac{u_{r} * d_{,} * \cos \alpha}{q_{w}}
\end{aligned}
$$

où $C$. et $d$. sont la concentration en air moyenne et la profondeur de référence à l'origine $(s=0)$.

A partir des mesures obtenues par STRAUB et LAMB (1956), IsACHENKo (1965), RAO et KoBUS (1971), CAIN (1978), Chanson (1988) et XI (1988), l'auteur a vérifié la validité de l'équation (3.3) (tabl. 2). La pente de ces courbes implique des valeurs de la vitesse limite d'ascension des bulles d'air $u_{r}$, comprises entre 0,2 et $41 \mathrm{~cm} / \mathrm{s}$. Ces valeurs correspondent à une valeur moyenne pour chaque série de mesures (fig. 7).

\section{Discussion}

Pour un liquide au repos, la vitesse limite d'ascension d'une bulle d'air est proportionnelle à $d_{b}^{2}$ pour des bulles sphériques rigides $\left(d_{b}<0,6 \mathrm{~mm}\right)$, et proportionnelle à $\sqrt{d_{b}}$ pour des bulles hémisphériques $\left(d_{b}>10 \mathrm{~mm}\right)$ 


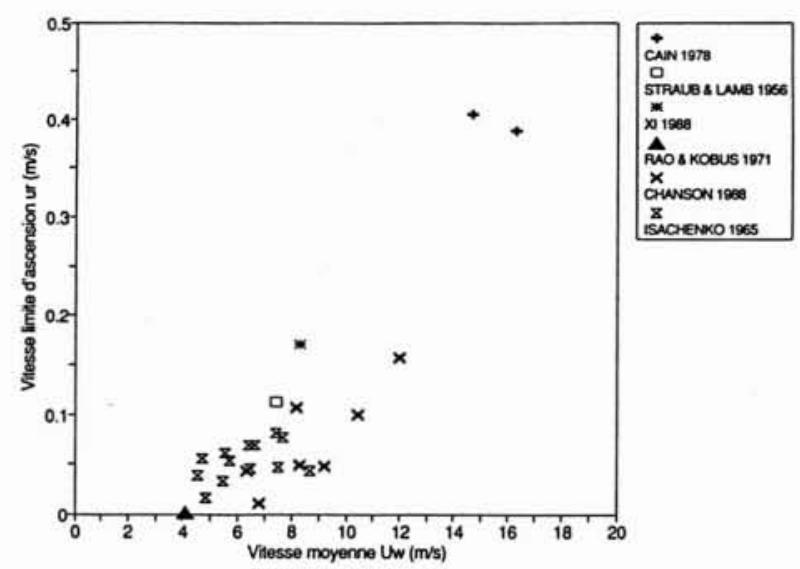

7. Vitesse limite d'ascension des bulles d'air : valeurs déduites de l'équation (3.3) - STRAuB and LAMB (1956) - ISACHENKO (1965) - RAO and KoBUS (1971) - CAIN (1978) Chanson $(1988)-\mathrm{XI}_{1}(1988)-u_{r}$ : vitesse limite d'ascension $(\mathrm{m} / \mathrm{s})-U_{w}$ : vitesse moyenne au début de la zone d'écoulement graduellement varié $(\mathrm{m} / \mathrm{s})$.

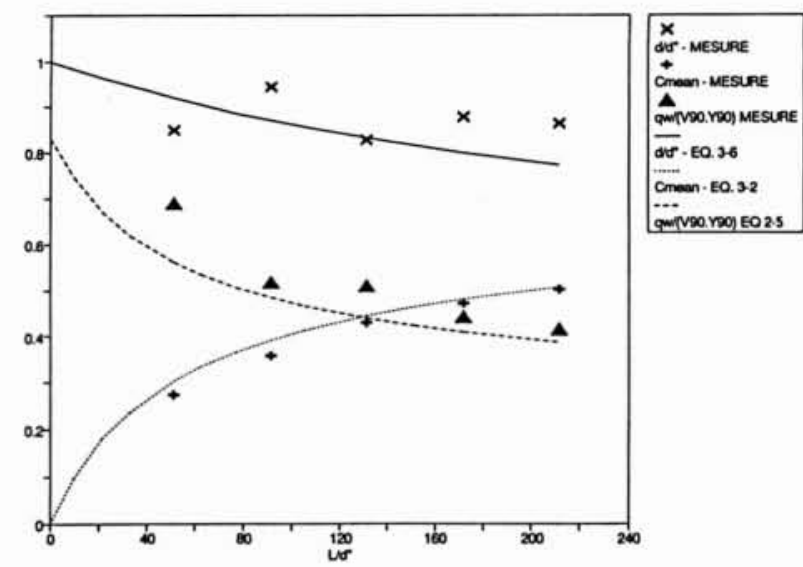

8. Entraînement d' air sur le coursier de l'évacuateur de crues du barrage d'Aviemore (Nouvelle-Zélande) $-\alpha=45^{\circ}-$ $q_{w}=2,16 \mathrm{~m}^{2} / \mathrm{s}-d^{*}=0,152 \mathrm{~m}-u_{r}=0,40 \mathrm{~m} / \mathrm{s}-k_{s}=$ $1 \mathrm{~mm}-$ CAIN (1978).
(Comolet 1979; Falvey 1980). La vitesse limite d'ascension des bulles d'air, dans un écoulement turbulent, est probablement différente des valeurs obtenues pour un liquide au repos, mais il est raisonnable de penser qu'elle augmente aussi avec la taille des bulles d'air.

Une étude fine des mesures obtenues par CAIN (1978) et CHANSON (1988) montre clairement que la taille des bulles d'air, et leur distribution à travers l'écoulement, diffère entre un modèle et un prototype (tabl. 3). Dans la plupart des cas, il n'est pas possible de respecter la vitesse d'ascension des bulles d'air, sur un modèle réduit, et il est donc difficile de modéliser correctement les phénomènes d'entraînement d'air.

Dans tous les cas, il est nécessaire d'étudier plus en détail l'interaction entre les bulles d'air, leur distribution et la turbulence dans la phase liquide de l'écoulement, pour comprendre les résultats obtenus sur la figure 7 .

\subsection{Equation de conservation de l'énergie}

Woop (1985) développa l'équation de conservation de l'énergie, pour un écoulement aéré, et montra que la charge totale $H$ est égale à :

$$
H=z+d * \cos \alpha+E * \frac{d}{2} * F r^{2}
$$

en supposant que la distribution des pressions est quasi hydrostatique, et où $E$ est le coefficient de correction d'énergie cinétique précédemment défini (équation (2.7)). Pour un écoulement graduellement varié, l'équation de conservation d'énergie devient :

$$
\frac{\mathrm{d}}{\mathrm{d} s} H=-S_{f}
$$

où $S_{f}$ est la pente de la ligne de charge :

$$
S_{f}=\frac{q_{w} * f}{8 * g * d^{2} *\left(\frac{D_{H}}{4}\right)} *\left(\frac{f_{e}}{f}\right)
$$

avec $f$ le coefficient de perte de charge pour un écoulement non-aéré. En combinant avec l'équation (3.4), on obtient (WOOD 1985; CHANSON 1989);

$$
\begin{aligned}
& \frac{\mathrm{d}}{\mathrm{d} s^{\prime}} d^{\prime}= \\
& =\frac{\sin \alpha *\left(1+d^{\prime} * \frac{\mathrm{d} \alpha}{\mathrm{d} s^{\prime}}\right)-S_{f}+E * \frac{d^{\prime}}{W^{\prime}} * \frac{F r_{*}^{2}}{d^{\prime 3}} * \frac{\mathrm{d} W^{\prime}}{\mathrm{d} s^{\prime}}}{\cos \alpha-E * \frac{F r_{*}^{2}}{d^{\prime 3}}}
\end{aligned}
$$

où $d=d^{*}$ à l'origine $(s=0), d^{\prime}=d / d_{\text {, }}, s^{\prime}=s / d_{\text {. }}$, $W^{\prime}=W / d_{\text {, }}$ et $F r_{.}=q_{w} / \sqrt{g * d_{*}^{3}}$. Pour un écoulement à surface libre, la profondeur de référence $d$. est la profondeur au point d'inception de l'entraînement d'air : $d_{.}=d_{1}$.

Les variations lentes des vitesses et des concentrations en air suggèrent que la pente de la ligne de charge $S_{f}$ et le coefficient $E$ peuvent être calculés, comme pour un écoulement uniforme, en utilisant la concentration en air moyenne locale $C_{\text {mean }}$ (WOOD 1985 ; CHANSON 1989).

\subsection{Discussion}

Les équations (3.2) et (3.6) fournissent un système de deux équations différentielles, à deux inconnues $d$ et $C_{\text {mean }}$, qui peut être résolu par une simple méthode numérique explicite.

La connaissance de la profondeur de référence $d$ et de la concentration en air moyenne $C_{\text {mean }}$ permet de 
calculer en tout point : $1^{\circ}$ la profondeur de référence $Y_{90}$ (équation (1.2)), $2^{\circ}$ le coefficient de perte de charge $f_{e}$ (équation (2.8)), $3^{\circ}$ la vitesse de référence $V_{90}$ (équation (2.5)), $4^{\circ}$ les constantes d'intégration $B^{\prime}$ et $G^{\prime}$ (tabl. 1), et $5^{\circ}$ les profils de vitesses et de concentrations en air.

Wood (1985) et Chanson (1989) ont utilisé ces équations pour simuler l'entraînement d'air pour le coursier de l'évacuateur de crues du barrage d'Aviemore, et sur le modèle de l'évacuateur de crues du barrage de Clyde. De même, l'auteur vérifia la validité de ces équations pour les mesures obtenues par STRAUB et LAMB (1956), IsACHENKO (1965), RAO et KobUS (1971), CAIN (1978), Chanson (1988) et XI (1988). Comme exemple, la figure 8 présente une comparaison entre les mesures expérimentales obtenues par CAIN (1978) et les équations (3.2), (3.6) et (2.5).

Les résultats obtenus sont bons mais l'auteur tient à souligner qu'ils dépendent critiquement des valeurs de la vitesse d'ascension des bulles d'air (tabl. 3). De plus les équations (3.2) et (3.6) ne sont valides que pour des écoulements graduellement variés, avec une distribution de pression quasi hydrostatique.

\subsection{Applications pratiques}

Ces calculs permettent de connaître en tout point du coursier les caractéristiques de l'écoulement, et nous indiquons ci-dessous trois applications pratiques:

$1^{\circ}$ En connaissant les profils des concentrations en air, en tout point du coursier, il est possible d'estimer la hauteur requise pour les murs latéraux du coursier.

$2^{\circ}$ Pour les calculs d'un saut de ski, et de la position d'un bassin de dissipation en aval d'un coursier, la méthode proposée fournit le profil des vitesses et leurs valeurs, à la base du tremplin, et ceci permet de calculer la portée théorique du jet. De plus, connaissant le taux d'aération à la base du tremplin, on pourra utiliser les calculs de ERVINE et FALVEY (1987), pour déterminer l'entraînement d'air au long du jet, ainsi que la dissipation d'énergie du jet dans le bassin de dissipation.

$3^{\circ}$ L'érosion par cavitation peut se développer à la surface du coursier, si la concentration en air près de cette surface est inférieure à $5-10 \%$, ce qui correspond à une concentration en air moyenne $C_{\text {mean }}$ inférieure à $30 \%$ (ChANSON 1989). Se basant sur l'expérience de l'US Bureau of Reclamation, FALVEY (1990) montre que le risque d'érosion par cavitation existe si le coefficient de cavitation, défini comme :

$$
\sigma=\frac{P-P_{v}}{\frac{1}{2} \rho_{w} * V^{2}}
$$

est inférieur à 0,20 . Aux portions du coursier où $\sigma<0,20$, on vérifiera que la concentration en air près de la surface du coursier est supérieure à $5-10 \%$, sinon il sera nécessaire d'introduire un dispositif d'aération (CHANSON 1989).

\section{Conclusion}

Pour des écoulements à surface libre, il est important de connaître la quantité d'air entraînée dans l'écoulement. La présence d'air dans l'écoulement augmente le volume de l'écoulement, réduit le coefficient de perte de charge mais aussi accélère le transfert d'oxygène et d'azote entre l'eau et l'air. De plus, la présence de petites quantités d'air, proches du coursier, permet de réduire, ou de stopper les dommages dus à l'érosion par cavitation.

Pour des écoulements uniformes avec entraînement d'air, les caractéristiques complètes de l'écoulement se déduisent à partir de la pente du coursier, du débit et du coefficient de perte de charge pour un écoulement nonaéré.

Pour un écoulement graduellement varié, on a présenté une analyse simple, basée sur l'équation de conservation de la masse pour l'air, et sur l'équation de conservation de l'énergie, qui fournit deux équations différentielles, pouvant être résolues numériquement. Ces équations permettent d'estimer, en tout point, les distributions de vitesses et de concentrations en air, ainsi que le taux d'aération de l'écoulement.

L'auteur souhaite souligner que ces résultats dépendent des valeurs estimées pour la vitesse limite d'ascension des bulles d'air, et pour la vitesse d'entraînement. De plus l'estimation du coefficient de perte de charge pour les écoulements aérés nécessite des études supplémentaires, en particulier pour mieux comprendre l'interaction entre les bulles d'air et la turbulence dans la phase liquide.

\section{Remerciements}

L'auteur tient à remercier : $1^{\circ} 1^{\prime}$ Ecole d'Ingénieurs de Génie Civil de l'Université de Queensland pour son support, $2^{\circ}$ son collègue, Dr. P. NIELSEN, pour ses suggestions constructives, et $3^{\circ}$ le Professor I.R. WooD qui incita l'auteur à s'intéresser à ces problèmes. 


\section{Appendice I. Tables de résultats}

1.

\begin{tabular}{|c|c|c|c|c|c|c|}
\hline $\begin{array}{c}\text { Pente } \\
\text { (degres) }\end{array}$ & $\begin{array}{l}\mathrm{C}_{\mathrm{e}} \\
\text { (i) }\end{array}$ & $\begin{array}{l}\mathrm{G}^{* *} \cos \alpha \\
\text { (II) }\end{array}$ & $\begin{array}{l}\text { B' } \\
\text { (II) }\end{array}$ & $\frac{q_{w}}{V_{90} \cdot Y_{90}}$ & M & E \\
\hline 7.5 & 0.1608 & 7.99952 & 0.003021 & 0.6880 & 1.029 & 1.075 \\
\hline 15.0 & 0.2411 & 5.74469 & 0.028798 & 0.6093 & 1.039 & 1.097 \\
\hline 22.5 & 0.3100 & 4.83428 & 0.071572 & 0.5540 & 1.033 & 1.085 \\
\hline 30.0 & 0.4104 & 3.82506 & 0.196353 & 0.4670 & 1.042 & 1.105 \\
\hline 37.5 & 0.5693 & 2.67484 & 0.620262 & 0.3350 & 1.061 & 1.148 \\
\hline 45.0 & 0.6222 & 2.40096 & 0.815675 & 0.3013 & 1.038 & 1.097 \\
\hline 60.0 & 0.6799 & 1.89421 & 1.353931 & 0.2409 & 1.107 & 1.249 \\
\hline 75.0 & 0.7209 & 157440 & 1.864181 & 0.2058 & 1.138 & 1.318 \\
\hline 0.0 & 0.000 & + infini & 0.00000 & 0.8571 (ail) & $1.021(\mathrm{cin})$ & $1.059 \mathrm{~cm}$ \\
\hline
\end{tabular}

Table 1 - Ecoulements uniformes : grandeurs caractéristiques des distributions de concentrations en air et de vitesses

Notes: (t) Mesures de STRAUB and ANDERSON (1958)

(ii) Calculés à partir des mesures de STRAUB and ANDERSON (III) Calculés pour $C_{e}=0$

2.

\begin{tabular}{|c|c|c|c|c|c|c|c|}
\hline Site & $\begin{array}{c}\text { Pente } \\
\text { (degrés) }\end{array}$ & $\begin{array}{c}q_{w} \\
\left(m^{2} / s\right)\end{array}$ & $\begin{array}{l}\mathrm{U}_{\mathrm{w}}(\mathrm{t}) \\
(\mathrm{m} / \mathrm{s})\end{array}$ & $\begin{array}{l}\mathrm{u}_{\mathrm{t}}(\mathrm{dl}) \\
(\mathrm{m} / \mathrm{s})\end{array}$ & $\begin{array}{c}\mathrm{k}_{\mathrm{s}} \\
\mathrm{mm}\end{array}$ & $\begin{array}{c}\mathbf{R} \\
\text { correl. }\end{array}$ & Commentaires \\
\hline Aviemore (III) & 45.0 & $\begin{array}{l}2.23 \\
3.16\end{array}$ & $\begin{array}{l}14.7 \\
16.3\end{array}$ & $\begin{array}{l}0.405 \\
0.389\end{array}$ & $\begin{array}{l}1.0 \\
1.0\end{array}$ & $\begin{array}{l}0.998 \\
0.981\end{array}$ & \multirow[t]{17}{*}{$\begin{array}{l}\text { Ecoulement a surface } \\
\text { libre }\end{array}$} \\
\hline St Anthony's fall (IV) & 30.0 & 0.396 & 7.4 & 0.112 & 0.05 & 0.988 & \\
\hline Meishan (V) & 52.5 & 0.320 & 8.3 & 0.170 & 0.07 & 0.995 & \\
\hline Rao et Kobus (VI) & 31.98 & 0.095 & 4.1 & 0.0025 & 0.91 & 0.339 & \\
\hline \multirow[t]{13}{*}{ Isachenko (VII) } & \multirow[t]{13}{*}{21.25} & 0.15 & 4.56 & 0.0386 & 0.1 & 0.966 & \\
\hline & & 0.25 & 5.47 & 0.0327 & 0.1 & 0.991 & \\
\hline & & 0.40 & 6.47 & 0.0456 & 0.1 & 0.983 & \\
\hline & & 0.60 & 7.48 & 0.0467 & 0.1 & 0.994 & \\
\hline & & 0.90 & 8.65 & 0.0429 & 0.1 & 0.844 & \\
\hline & & 0.25 & 4.85 & 0.0162 & 3.0 & 0.682 & \\
\hline & & 0.40 & 5.74 & 0.0528 & 3.0 & 0.989 & \\
\hline & & 0.60 & 6.63 & 0.0687 & 3.0 & 0.991 & \\
\hline & & 0.90 & 7.66 & 0.0764 & 3.0 & 0.991 & \\
\hline & & 0.25 & 4.71 & 0.0556 & 7.0 & 0.903 & \\
\hline & & 0.40 & 5.57 & 0.0614 & 7.0 & 0.945 & \\
\hline & & 0.60 & 6.43 & 0.0691 & 7.0 & 0.903 & \\
\hline & & 0.90 & 7.44 & 0.0816 & 7.0 & 0.928 & \\
\hline \multirow{7}{*}{$\begin{array}{l}\text { Modèle du Clyde dam } \\
\text { (VII) }\end{array}$} & \multirow[t]{7}{*}{52.33} & 0.212 & 8.2 & 0.106 & 0.1 & $0.9 \times 5$ & \multirow{7}{*}{ un aéraleur } \\
\hline & & 0.396 & 12.0 & 0.157 & 0.1 & 0.567 & \\
\hline & & 0.345 & 10.5 & 0.100 & 0.1 & 0.714 & \\
\hline & & 0.304 & 9.2 & 0.048 & 0.1 & 0.639 & \\
\hline & & 0.273 & 8.3 & 0.049 & 0.1 & 0.867 & \\
\hline & & 0.210 & 6.4 & 0.044 & 0.1 & 0.977 & \\
\hline & & 0.429 & 6.8 & 0.011 & 0.1 & 0.685 & \\
\hline
\end{tabular}

Table 2 - Vitesses limites d'ascension des bulles d'air : valeur calculées $q_{w}$ débit par mètre de largeur $-U_{w}$ vitesse moyenne - $u_{r}$ vitesse limite d'ascension $k_{s}$ rugosité $-R$ coefficient de corrélation

Note: (1) Vitese moyenne au début de la zone đ'écoulement graduellement varié :

$U_{w}=q_{w} / d_{I}$ (écoulement à surface libre) $\quad U_{w}=q_{w} / d$ e (écoulement en aval d'un aérateur) $\begin{array}{lll}\text { (ii) Equation (3-3) (III) CAIN (1978) } & \text { (IV) STRAUB et LAMB (1956) }\end{array}$

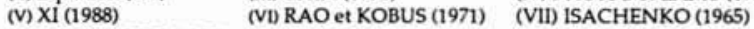

(vii) CHANSON (1988)

3.

\begin{tabular}{|c|c|c|c|c|c|}
\hline Site & $\begin{array}{c}\begin{array}{c}\text { Pente } \\
\text { (degrts) }\end{array} \\
\end{array}$ & $\begin{array}{c}q_{w} \\
\left(\mathrm{~m}^{2} / \mathrm{s}\right)\end{array}$ & $\begin{array}{l}U_{w}(1) \\
(\mathrm{m} / \mathrm{s})\end{array}$ & $\begin{array}{l}\text { Taille des } \\
\text { bulles } d_{b}\end{array}$ & Commentaires \\
\hline Aviemore (I) & 45.00 & 2.23 et 3.16 & $14.7 \times 16.3$ & $5220 \mathrm{~mm}$ & $\begin{array}{l}\text { Prototype. Coursier de grande } \\
\text { largeur. }\end{array}$ \\
\hline $\begin{array}{l}\text { Modele du Clyde dam } \\
\text { (III) }\end{array}$ & 52.33 & $0.20 \times 0.40$ & $6.4 \times 12.0$ & $0.3 \mathrm{a} 4 \mathrm{~mm}$ & $\begin{array}{l}\text { Modele. Ecoulement en aval } \\
\text { d'un atrateur. } W=0.25 \mathrm{~m} \text {. }\end{array}$ \\
\hline St Anthony's fall (IV) & $\ldots$ & $0.136 \mathrm{a} 0.793$ & $\ldots$ & $0.7 \mathrm{a} 2.7 \mathrm{~mm}$ & $\begin{array}{l}\text { Moddle de STRAUB } \\
\text { ANDERSON. } W=0.457 \mathrm{~m} .\end{array}$ \\
\hline
\end{tabular}

Table 3 - Diamètre moyen des bulles $d$ 'air : $d_{b}$ diamètre des bulles $d$ 'air

Note : (f) Vitese moyenne au début de la zone d'écoulement graduellement varié :

$U_{w}=q_{w} / d_{I}$ (tcoulement a surface libre) $\quad U_{w}=q_{w} / d$. (tcoulement en aval d'un aérateur) (i) CAIN (1978) (III) CHANSON (1988)

$U_{w}=q_{w} / d .($ ecoulement en aval
(IV) GULLVER et al. (1990) 


\section{Appendice II. Références}

AIVAZYAN, O.M. (1986). "Stabilized Aeration on Chutes." Gidrotekhnicheskoe Stroitel'stvo, No. 12, pp. 33-40 (Hydrotechnical Construction, 1987, Plenum Publ., pp. 713-722).

CAIN, P. (1978). "Measurements within Self-Aerated Flow on a Large Spillway." Research Report No. 78-18, Univ. of Canterbury, New Zealand.

CAIN, P., and WOOD, I.R. (1981). "Measurements of Self-aerated Flow on a Spillway." Jl. Hyd. Div., ASCE, 107, HY11, pp. 1425-1444.

CHANSON, H. (1988). "Study of Air Entrainment and Aeration Devices on Spillway Model." Research Report No. 88-8, Univ. of Canterbury, New Zealand.

CHANSON, H. (1989). "Etude des phénomènes d'entraînement d'air. Application aux évacuateurs de crues." Jl. La Houille Blanche, No. 6, 1989, pp. 441-462.

COLEMAN, N.L. (1981). "Velocity Profiles with Suspended Sediment." Jl. of Hyd. Res., IAHR, Vol. 19, No. 3, pp. 211-229.

COMOLET, R. (1979). "Vitesse d'ascension d'une bulle de gaz isolée dans un liquide peu visqueux." $/ l$ de Mécanique Appliquée, Vol. 3, No. 2, pp. 145-171.

ELATA, C., and IPPEN, A.T. (1961). "The Dynamics of Open Channel Flow with Suspensions of Neutrally Buoyant Particles." Mass. Inst. of Techn. Hydrodynamic Lab. Tech. Report, Dept. of Civil and Sanitary Engrg. Techn., No. 45, 69 pp.

ERVINE, D.A., and FALVEY, H.T. (1987). "Behaviour of Turbulent Water Jets in the Atmosphere and in Plunge Pools." Proc. Instn Civ. Engrs, Part 2, 1987, 83, Mar., pp. 295-314.

FALVEY, H.T. (1980). "Air-Water Flow in Hydraulic Structures." USBR Eng. Monograph, No. 41, Denver, Colorado, USA.

FALVEY, H.T. (1990). "Cavitation in Chutes and Spillways." USBR Eng. Monograph, No. 42, Denver, Colorado, USA.

GULLIVER, J.S., THENE, J.R., and RINDELS, A.J. (1990). "Indexing Gas Transfer in Self-Aerated Flows." /l of Environm. Engrg., ASCE, Vol. 116, No. 3, pp. 503-523.

HARTUNG, F., and SCHEUERLEIN, H. (1970). "Design of Overflow Rockfill Dams." 10th ICOLD Congress, Montréal, Canada, Q. 36, R. 35, pp. 587-598.

ISACHENKO, N.B. (1965). "Effect of Relative Roughness of Spillway Surface on Degree of Free-Surface Flow Aeration." Izv. VNIIG, Vol. 78, pp. 350-357 (in Russian).

JEVDJEVICH, V., and LEVIN, L. (1953). "Entrainment of Air in flowing Water and Technical Problems connected with it." Proc. of 5th I.H.R. Congress, Minneapolis, USA, 1953, pp. 439-454.

KELLER, R.J., and RASTOGI, A.K. (1977). "Design Chart for Predicting Critical Point on Spillways." $/ /$ of $H y d$. Div., Proc. ASCE, Vol. 103, No. HY12, pp. 1417-1428.

KILLEN, J.M. (1968). "The Surface Characteristics of Self-Aerated Flow in Steep Channels." Ph.D. thesis, University of Minnesota, Minneapolis, USA.

KNAUSS, J. (1979). "Computation of Maximum Discharge at Overflow Rockfill Dams (a comparison of different model test results)." 13th ICOLD Congress, New Delhi, India, Q. 50, R. 9, pp. 143-159.

MAY, R.W.P. (1987). "Cavitation in Hydraulic Structures : Occurrence and Prevention." Hydraulics Research Report, No. SR 79, Wallingford, UK.

RAO, N.S.L., and KOBUS, H.E. (1971). "Characteristics of Self-Aerated Free-Surface Flows." Water and Waste Water / Current Research and Practice, Vol. 10, Eric Schmidt Verlag, Germany.

STAUB, L.G., and ANDERSON, A.G. (1958). "Experiments on Self-Aerated Flow in Open Channels." $J l$ of Hyd. Div., Proc. ASCE, Vol. 84, No. HY7, paper 1890.

STRAUB, L.G., and LAMB, O.P. (1956). "Studies of Air Entrainment in Open-Channel Flows." Trans. ASCE, Vol. 121, pp. 30-44.

VANONI, V.A. (1946). "Transportation of suspended sediment in water." Trans. ASCE, Vol. 111, pp. 67-133.

WILHELMS, S.C., and GULLIVER, J.S. (1989). "Self-Aerating Spillway Flow." National Conference on Hydraulic Engineering, ASCE, New Orleans, USA, M.A. PORTS editor, pp. 881-533.

WOOD, I.R. (1983). "Uniform Region of Self-Aerated Flow." $l l$ Hyd. Eng., ASCE, Vol. 109, No. 3, pp. 447-461.

WOOD, I.R. (1984). "Air Entrainment in High Speed Flows." Symp. on Scale Effects in modelling Hydraulic Structures, IAHR, Esslingen, Germany, H. KOBUS editor, paper 4.1.

WOOD, I.R. (1985). "Air Water Flows." 21st IAHR Congress, Melbourne, Australia, Keynote address, pp. 1829.

XI, Ruze (1988). "Characteristics of Self-Aerated Flow on Steep Chutes." Intl Symp. un Hydraulics for High Dams, IAHR, Beijing, China, pp. 68-75. 